\title{
Nikolai Anichkov y los cien años de la hipótesis sobre el colesterol y la aterogénesis
}

\author{
Nikolai Anichkov and one hundred \\ years of the hypothesis about \\ cholesterol and atherogenesis
}

\begin{abstract}
The relationship of cholesterol to atherogenesis already fulfilled one hundred years. The merit of this important discovery is attributed to a Russian military doctor, Nikolai Anichkov, who using rabbits as experimental model, was able to show the accumulation of "lipoids" when he fed animals with a diet rich in cholesterol. His experimental observations were criticized by many researchers, since he used an animal that is not carnivorous, so it was an inappropriate model. Anichkov published very few scientific articles, and all of them in Russian language. Only in 1933 he could make public his work to the Western world by publishing a review on his scientific work in English. Was only in 1984 when the scientific community recognized the merit of his research, which was pioneer in the understanding that we have today about the relationship of artherogenesis and cholesterol. Key words: Nikolai Anichkov, cholesterol, atherogenesis.
\end{abstract}

\begin{tabular}{r} 
Alfonso Valenzuela B. (1,3) \\
Miguel Ángel Rincón (1) \\
Rodrigo Valenzuela (1,2) \\
\hline (1) Laboratorio de Lípidos y Antioxidantes, \\
Instituto de Nutrición yecnología de los Alimentos (INTA), \\
Universidad de Chile, Santiago, Chile. \\
(2) Departamento de Nutrición, Facultad de Medicina, \\
Universidad de Chile, Santiago, Chile. \\
(3) Escuela de Nutrición, Facultad de Medicina, \\
Universidad de los Andes, Santiago, Chile. \\
Dirigir la correspondencia a: \\
Profesor \\
Alfonso Valenzuela B. \\
correo electrónico: avalenzu@inta.uchile.cl
\end{tabular}

Este trabajo fue recibido el 30 de Noviembre de 2015 y aceptado para ser publicado el 11 de Agosto de 2016.

\section{EL ORIGEN DE LA HIPÓTESIS}

En la era de la lipidómica, proteómica y la metabolómica, es difícil creer que la asociación del colesterol con la aterogénesis ya tiene más de cien años. Así es y, por lo demás, es una interesante historia científica. Nikolai N. Anichkov (o Anitshkow, en versión alemana) fue un investigador médicomilitar formado en la más conservadora tradición de la Rusia Zarista nacido en 1885 en San Petersburgo, Rusia. Graduado como médico comenzó a servir en 1903 en la Academia Médica Militar Imperial de San Petersburgo y combinaba sus deberes militares con su curiosidad científica realizando investigación como patólogo experimental. La observación de muestras de anatomía patológica provenientes de pacientes fallecidos en el Hospital General de San Petersburgo, motivó su curiosidad por entender por qué en los pacientes de mayor edad observaba lesiones en los grandes vasos, especialmente en aquellos con bifurcaciones, en las cuales identificó la presencia de colesterol, sustancia descubierta por el anatomista francés Poulletier de la Salle (1719-1788), quien en 1769 aisló un compuesto de carácter "aceitoso" (según su propia definición) desde la vesícula biliar de cadáveres (1). Quien redescubrió el colesterol años después fue el gran químico, también francés, Michel-Eugéne Chevreul (1786-1889), reconocido como el "padre" del conocimiento sobre las materias grasas. El producto que separó de la bilis humana lo llamó "colesterina" aunque su función metabólica no era entendida (2).

En aquella época las lesiones de los vasos sanguíneos se identificaban como "lipoides" y se asociaban principalmente como "efectos de la vejez", hipótesis apoyada por muchos anatomistas y patólogos. Motivado por su vocación experimentalista Anichkov decidió investigar el origen de las lesiones asociadas a los "lipoides". Entre 1903 y 1905 la hipótesis más preponderante sobre el origen de estas lesiones era la de Ilia Metchnikow (1845-1916), también médico e investigador ruso, quien proponía que estas lesiones eran el resultado de un excesivo consumo de proteínas de origen animal. Su hipótesis no tenía respaldo científico pero era aceptada debido al prestigio de quien la proponía, Metchnikow es más conocido como el "padre de los probióticos". Siguiendo esta hipótesis, Anichkov decidió alimentar conejos con leche, huevos, yema de huevos y con diferentes proteínas de origen animal. En todos los protocolos experimentales encontró lipoides, aunque no pudo identificar qué tipo de proteína los producía. Finalmente, no convencido con la hipótesis de Metchnikow, quien ya en aquella época era un respetable Premio Nobel, Anichkov animó a uno de sus estudiantes, Semen S. Chalatov, para primero purificar colesterol a partir de la yema de huevos, 
disolverlo en aceite de girasol (o maravilla) y administrárselo a conejos durante un período de 3 semanas. Finalizados los protocolos pudo observar que en todos los animales aparecían alteraciones, lesiones que iban desde estrías grasas hasta grandes acúmulos de "lipoides" y en todas ellas identificó la presencia de colesterol.

\section{DUDAS SOBRE LA VALIDEZ \\ DEL MODELO EXPERIMENTAL}

Sin embargo, quedaba la duda si lo observado en los conejos tendría validez en otros animales, particularmente en los humanos. Al ser un animal vegetariano, el conejo prácticamente no consume colesterol y todo el que necesita para sus procesos metabólicos debe provenir de su propia síntesis. Curiosamente, Anichkov no realizó experimentos con otros animales lo cual resultó un "error afortunado" porque si hubiese utilizado un animal carnívoro como el perro, no habría observado "lipoides" ya que este animal, como todos los verdaderos carnívoros (a excepción de nosotros los humanos) (3) elimina todo el colesterol excedente por vía biliar y este no se acumula en sus arterias, en otras palabras, no sufren de arterioesclerosis. En 1913 publicó su más reconocido trabajo sobre la relación del colesterol con las lesiones vasculares. (4) Insistió en el uso de los conejos, e hizo oídos sordos a la crítica de sus colegas sobre la valides de su modelo experimental. Cuidadosamente se dedicó a observar la anatomía de las lesiones vasculares. En 1933 publicó una revisión sobre sus observaciones y también la de otros investigadores sobre las características anátomo-patológicas de las lesiones derivadas de exceso de colesterol circulante (5).

Algunos laboratorios trataron de confirmar el hallazgo de Anichkov con éxito cuando utilizaron conejos o cerdos de guinea, como lo demostró C. Bailey en la Universidad de Stanford en 1915 (6). Sin embargo, otros investigadores al utilizar ratas o perros, no llegaron a conclusiones similares ya que la administración de altas cantidades de colesterol a estos animales no generaba "lipoides". Hoy sabemos que estos animales pueden eliminar muy eficientemente el colesterol a través de la bilis y/o transformarlo en sales biliares, algo que no ocurre en el conejo. Estas observaciones, desacreditaron los hallazgos de Anichkov y se consideró que el conejo no era un buen modelo para el estudio de los efectos metabólicos del colesterol. Post revolución rusa, Anichkov se transformó, voluntaria (o involuntariamente), en un disciplinado comunista ya que incluso llegó a ser consultor científico de Joseph Stalin. Así fue también como se generó el cerco político y de aislamiento que caracterizó a la Rusia Soviética (URSS), que sepultó en el olvido del mundo occidental la importante observación del investigador. Varios años después, en 1945 Steiner and Kendall (7) demostraron que el tratamiento de perros con tiouracilo, que sabemos que bloquea la exposición de receptores de LDL, y la posterior administración de colesterol, incrementaba la presencia de este metabolito en la sangre de los perros e inducía lesiones ateroscleróticas. Esto es, la observación de Anichkov pero ahora en un animal carnívoro. Entonces, bloqueando la utilización celular del colesterol era posible producir lesiones vasculares, algo que nuestro investigador no supo intuir o no sabía cómo demostrarlo. Otro motivo de crítica al trabajo de Anichkov fue que los niveles de colesterol que alcanzaba en sus conejos eran de 800 a 1000 mg/dL de sangre o más, algo que no ocurre en los humanos (salvo en condiciones genéticas de hipercolesterolemia familiar homocigota), lo cual también desacreditaba la interpretación de sus resultados. Anichkov realizó experimen- tos posteriores en los que demostró que cantidades menores de colesterol también inducían lesiones en los conejos. Como publicó en lenguaje ruso sus hallazgos, fueron prácticamente desconocidos en el mundo occidental. Su única publicación en alemán es la ya mencionada, publicada en 1913. Quizás, el movimiento comunicacional que produjo la pre-segunda guerra mundial (pactos entre alemanes y rusos, pactos entre franceses e ingleses e incluso contactos entre ingleses y rusos) abrió alternativas de comunicación entre Rusia y la Europa Occidental. Es así como Anichkov pudo publicar su trabajo de 1933 en inglés en el cual describió todos los hallazgos de su laboratorio, ahora en un lenguaje común para los científicos. Esta publicación despertó la curiosidad y el interés de los patólogos y experimentalistas.

Un motivo de controversia para los resultados de Anichkov fue que en aquella época, comienzo del siglo 20, la arterosclerosis era considerada una enfermedad o anomalía funcional sólo típica de la vejez, esto es avanzaba crónicamente etapa por etapa en la medida de los años, entonces ¿Cómo era posible reproducir la enfermedad en animales jóvenes y en un tiempo tan corto, algo que normalmente demora décadas en el humano?. En la visión de aquella época parecía ilógico. Los estudios posteriores de muchos investigadores demostraron finalmente que una condición de hipercolesterolemia crónica, aún en animales jóvenes, se relacionaba con la aterogénesis.

\section{SE CONSOLIDA Y ACEPTA}

\section{LA HIPÓTESIS DE ANICHKOV}

El primer norteamericano en reconocer la validez e importancia de los estudios de Anichkov fue el patólogo John Gofman. En un artículo publicado en Science en 1950, Gofman comunicó que al separar el suero de conejos alimentados con colesterol, según los protocolos de Anichkov, mediante el uso de la ultracentrífuga, un instrumento de separación analítica revolucionario en la época, el colesterol se separaba en dos fracciones claramente identificables (8). Una fracción prácticamente flotaba en la superficie del suero después de la ultracentrifugación y era una mezcla de proteínas, fosfolípidos y colesterol, esto es una lipoproteína. Gofman la denominó "low density lipoprotein" o "lipoproteína de baja densidad", y que no era otra cosa que la lipoproteína conocida como LDL. La otra fracción, también de carácter lipoproteico, era de mayor densidad, por lo cual fue denominada "high density lipoprotein" o "lipoproteína de alta densidad", y corresponde a la lipoproteína que identificamos como HDL. Gofman, también observó que mientras en los conejos no alimentados con colesterol, la mayor parte del colesterol era transportado en las HDL, en los conejos hipercolesterolémicos el colesterol era mayoritariamente transportado por las recientemente descubiertas LDL. Posteriormente, Gofman y su grupo ultracentrifugaron el suero de 104 hombres con antecedentes previos de arteriosclerosis y de infarto. En 101 de ellos encontraron elevado el colesterol contenido en las LDL (colesterol-LDL).

Resultados similares fueron obtenidos a partir de mujeres con antecedentes de enfermedad coronaria. La publicación del artículo de Gofman abrió, literalmente, los ojos de la comunidad científica y médica ante los peligros del colesterol de la dieta. Otro hallazgo de Gofman, fue resistido en aquella época por algunos cardiólogos, se refiere a que no es el colesterol total el indicador de riesgo cardiovascular, sino el colesterolLDL. Los resultados de Gofman recibieron una publicidad muy amplia en los medios de comunicación no especializa- 
dos, aunque numerosos cardiólogos de la época se negaban a aceptar la idea que solo un tipo de colesterol unido a una lipoproteína fuese la sustancia responsable de la aterogénesis. Sin embargo, la dificultad técnica derivada de la utilización de la ultracentrífuga, en aquella época un instrumento de alto costo, limitó un mayor progreso en el conocimiento del rol de los distintos tipos de lipoproteínas y de su contenido de colesterol en la aterogénesis, con lo cual el progreso sobre el tema fue muy lento. Durante la década de 1960 y 1970 se desarrollaron nuevos métodos económicos y al alcance de la mayoría de los laboratorios, como es la electroforesis o los kits de inmunoprecipitación, para la separación e identificación de las lipoproteínas del suero sanguíneo, con lo cual fue posible la realización de numerosos estudios epidemiológicos que "redescubrieron" el peligro potencial del colesterol asociado a las LDL.

En forma paralela, pero absolutamente independiente de los estudios de Gofman, Lawrence Kinsell, un médico clínico californiano, descubrió en 1952 que la alimentación con vegetales, asociada a una disminución de la ingesta de productos animales, producía una disminución del colesterol plasmático, particularmente del colesterol-LDL (9). Este estudio fue confirmado posteriormente por otro grupo de investigadores encabezados por E. H. Ahrens (10) quienes, además, asociaron al consumo de grasas insaturadas con la reducción del colesterol plasmático. Lamentablemente, el desenlace de los estudios de Kinsell fue dramático. Kinsell trabajaba en un hospital del condado de Alameda, California, cuya disponibilidad de camas y de recursos para sus estudios nutricionales era muy limitada. Diariamente recibía el asedio y la crítica de sus colegas por el uso "inútil" de camas de uso clínico en sus estudios. Fue recriminado por la dirección del hospital. Profundamente deprimido por la situación, cierto día de 1953, Kinsell Ilamó a su secretaria para que acudiera a su casa, al llegar a esta encontró un espectáculo desolador, Kinsell y su esposa se habían suicidado ingiriendo cianuro (otro caso de asedio como el ocurrido con Alan Turing en 1954). Probablemente debido a este trágico final, nadie recuerda a Kinsell como el primero que asoció el consumo de grasas insaturadas con la reducción del colesterol plasmático.

\section{EL RECONOCIMIENTO DE \\ LA COMUNIDAD CIENTÍFICA}

El reconocimiento al trabajo pionero de Anichkov, e indirectamente de Gofman y Kinsell, llegó finalmente en 1958, a través de un artículo publicado en la revista Circulation por William Dock (11), quien en aquella época era el director del Departamento de Patología de la Facultad de Medicina de la Universidad de Stanford. No fue la comunicación de un trabajo de investigación científica, sino un editorial que reivindicaba el olvido al trabajo original de Anichkov y otorgaba el debido reconocimiento a los aportes de Gofman y del olvidado Kinsell. La conclusión de este visionario editorial fue que el colesterol de la dieta, y la composición de la dieta, desempeñan un papel fundamental en el mayor o menor desarrollo de la ateromatosis en las arterias tanto grandes como pequeñas. El resto de la historia del colesterol ha seguido un desarrollo vertiginoso, coronado, sin lugar a dudas, por el descubrimiento de los investigadores de la Universidad de Texas, en Dallas, USA, Michael Brown y Joseph Goldstein en 1983 del receptor celular de las LDL y del control intracelular que determina los niveles plasmáticos de colesterol-LDL y su relación con la aterogénesis (12).

¿Merecía Anichkov el Premio Nobel de Medicina? Quizás sí, pero los tiempos no fueron los correctos. Anichkov nació en 1885, publicó su primer trabajo en 1913, pero el reconocimiento de su visionaria investigación otorgado por la Academia de Cardiología Americana en 1984 y que lo habría hecho merecedor del Premio, ocurrió veinte años después de su muerte (1964). El Premio Nobel no se otorga en forma póstuma. La imagen muestra una fotografía del Dr. Anichkov cuando era teniente general del ejército soviético, 1945.

\section{RESUMEN}

La relación del colesterol con la aterogénesis ya cumplió cien años. El mérito de este importante descubrimiento lo tiene un médico militar ruso Nikolai Anichkov, quien utilizando conejos como modelo experimental, pudo demostrar la acumulación de "lipoides" cuando los alimentaba con una dieta rica en colesterol. Sus observaciones fueron criticadas por numerosos investigadores ya que utilizaba un animal

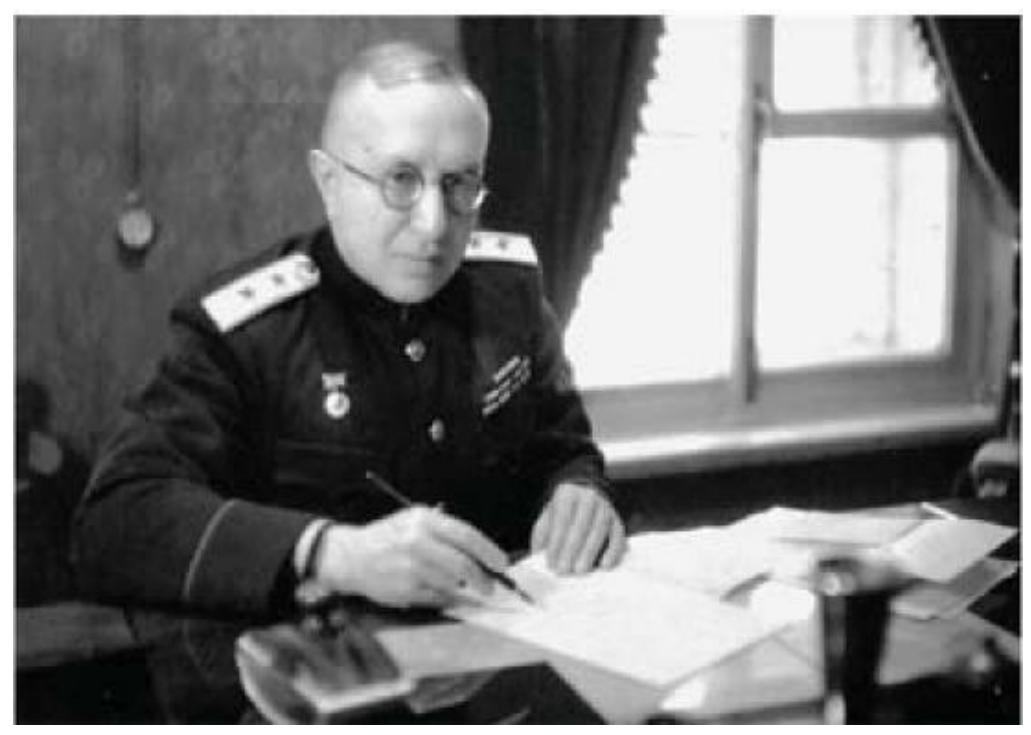

Dr. Nicolai Anichkov

(1885-1964) 
que no es carnívoro, por lo cual era un modelo inadecuado. Anichkov publicó muy pocos trabajos científicos y todos ellos en idioma ruso. Solo en 1933 pudo hacer público su trabajo al mundo occidental al publicar en inglés una revisión sobre su trabajo científico. Tardíamente, en 1984, se reconoció por parte de la comunidad científica el mérito de su investigación, la que fue pionera en la comprensión que tenemos hoy día sobre la enfermedad arterioesclerótica y el colesterol.

Palabras clave: Nikolai Anichkov, colesterol, aterogenesis.

\section{BIBLIOGRAFÍA}

1. Valenzuela $A$, Morgado N. Fats and oils in human nutrition: A historical overview. Rev Chil Nutr. 2005; 32: 88-94.

2. Valenzuela A, Morgado N. Brief history of the relationship between cholesterol and cardiovascular diseases. Rev Chil Nutr. 2006; 33: 130-134.

3. Valenzuela A. Why we eat what we eat? Rev Chil Nutr. 2011; 38: 198-209.

4. Anitshkow N N, Chalatov S. Ueber experimentelle Cholesterinsteatose und ihre Bedeutung fur die Entstehung einiger pathologischer Prozesse. Zentralbl Allg Pathol. 1913; 24: 1-9.

5. Anitschkow N N. Experimental atherosclerosis in animals. En: Arterioesclerosis, E V Cowdry Ed. Macmillan, New York, 1933: 271-322.

6. Bailey $\mathrm{CH}$. Observations on cholesterol-fed guinea pigs. Exp Biol Med. 1915; 13: 60-62.

7. Steiner A, Kendall F. Atherosclerosis and arteriosclerosis in dogs following ingestion of cholesterol and thiouracil. Arch Pathol.1946; 42: 433-44.

8. Gofman. J. W. The role of lipids and lipoproteins in arteriosclerosis. Science. 1950, III, 167-69.

9. Kinsell. L. W. Dietary modification of serum cholesterol and phospholipids levels. J Clin Endocrinol 1952; 12: 909-13.

10. Ahrens, E. H., Blankenhorn, D. H., Tsaltes, T. T. Effect of serum lipids of substituting plant for animal fat in diet. Proc Soc Exp Biol Med. 1952; 86: 872-879.

11. Dock. W. Research in arteriosclerosis- the First Fifty years, editorial, Ann Int Med. 1958; 49: 699-700.

12. Brown, M. S. Goldstein, J. L. Lipoprotein receptors in the liver. J Clin Invest. 1983; 72: 743-51. 